\title{
The World Neutron Monitor Network as a tool for the study of solar neutrons
}

\author{
I. G. Usoskin ${ }^{1}$, G. A. Kovaltsov ${ }^{1}$, H. Kananen ${ }^{2}$, P. Tanskanen ${ }^{2}$ \\ ${ }_{1}^{1}$ A.F. Ioffe Physical-Technical Institute, 194021 St.Petersburg, Russia \\ ${ }^{2}$ University of Oulu, Dept. of Physical Sciences, Linnanmaa, FIN-90570 Oulu, Finland
}

Received: 11 March 1996 / Revised: 11 November 1996 / Accepted: 26 November 1996

\begin{abstract}
The use of the World Neutron Monitor Network to detect high-energy solar neutrons is discussed in detail. It is shown that the existing network can be used for the routine detection of intense sporadic solar-neutron events whenever they occur. A technique is suggested involving the weighted summation of responses of separate monitors to solar neutrons. It is demonstrated that the use of this method improves the significance of solar-neutron event detection. Different results of the simulation of the neutron-monitor sensitivity to solar neutrons have been tested with respect to their application for practical use. It is shown that the total number of neutrons with energy above $300 \mathrm{MeV}$ injected from the Sun during a solar flare can be estimated directly from the time-integrated neutronmonitor response to solar neutrons without any model assumptions. The estimation technique has been developed.
\end{abstract}

\section{Introduction}

Ground-based neutron monitors (NMs) are widely used for registration of the nucleon component of cosmic rays in the Earth's atmosphere. An NM detects, with high efficiency, secondary nucleons produced by cosmic rays in the atmosphere. Throughout the paper, we use the words "NM detects galactic/solar particles" in the sense that we consider the system NM/atmosphere as one entity. At present, there is a worldwide network consisting of about 70 cosmic-ray stations equipped with NMs of different types. In this paper the network is called the World Neutron Monitor Network. There are two main types of NMs in operation: the IGY monitor and the NM64 monitor (Hatton, 1971). NMs detect the Galactic component of cosmic rays (GCR). In addition, the NM can detect solar cosmic rays (SCRs) with energies above several hundred $\mathrm{MeV} /$ nucleon. During the last three solar cycles, more than 50 ground-level enhancement (GLE) events caused by SCRs were observed by the World Neutron Monitor Network (Shea and Smart, 1990; Stoker, 1994).

It is very important for solar-physics research that the network can detect high-energy neutrons produced in the solar atmosphere during solar flares (e.g. Takahashi, 1989; Debrunner, 1994). Such neutrons are produced in nuclear reactions of high-energy particles with energies from several hundred $\mathrm{MeV} /$ nucleon to several $\mathrm{GeV} /$ nucleon with the matter of the solar atmosphere. Therefore, observations of solar neutrons offer a unique opportunity to study energetic nuclear processes as well as particle acceleration processes occurring during solar flares. Today, about ten reliably detected solar-neutron events (SNEs) are known. Two of the events were observed by several NMs during the solar flares of 3 June 1982 (Debrunner et al., 1983; Efimov et al., 1983; Iucci et al., 1984) and 24 May 1990 (Shea et al., 1991; Debrunner et al., 1993; Kovaltsov et al., 1993).

The goal of the present paper is to study the feasibility of the World Neutron Monitor Network as a tool for the investigation of high-energy solar neutrons. Unfortunately, the simulations of NM sensitivity to solar neutrons carried out by different research groups differ significantly from each other (see Sect. 5). Moreover, at present there are no practical ways to calibrate the atmosphere/NM system. Hereafter, the sensitivity concept includes both the instrument and the atmosphere above it. In the present paper, we tested the sensitivity by means of SNEs which were detected with high significance and reliability by different NMs. We also used data of neutrons and $\gamma$-rays observed by spacecraft in the interplanetary space. The use of these data allows one to estimate the neutron flux before it enters the Earth's atmosphere and facilitates a comparison with the system's response.

In Sect. 2 we discuss some special features of solarneutron detection by ground-based neutron monitors.

Further, in Sect. 3, the extraction of a weak signal of information from a high background "noise" is studied with particular emphasis on neutron monitors. Empir- 
ical expressions, which describe angular and altitude dependencies of the sensitivity of a neutron monitor to solar neutrons, are studied on the basis of observations of the neutron event of 24 May 1990. It is shown that the expression given by Kovaltsov et al. (1993) is in good agreement with observational records, and that the current NM network can be used for continous monitoring of solar neutron events.

The utilisation of useful information not only from a single monitor, but from the whole NM network as well, offers an improvement of the means for the detection of SNEs. In Sect. 4 the technique of weighted summation applied to NM responses is suggested. It is shown that the use of the method improves the significance of the detection of SNEs.

The results of the sensitivity simulation carried out by different groups differ from each other in both integral normalisation and the energetic dependence of the sensitivity in the energy range below $300 \mathrm{MeV}$. In Sect. 5 we make a test of the normalisation of the NM sensitivity by means of the solar flare of 3 June 1982 and show that the results by Debrunner et al. (published in Chupp et al., 1987) for the IGY-type monitor and by Efimov and Terekhov (1988) for the NM64 monitor are in a reasonable accordance with the observations. However, a new numerical simulation of the atmospheric nucleon cascade processes is still apparent. Although the question about the sensitivity of an NM to neutrons with energy below several hundred $\mathrm{MeV}$ is still open, it is shown in Sect. 6 that the main contribution to the NM response is due to neutrons with energy above $300 \mathrm{MeV}$.

Also in Sect. 6 of the paper we study the relationships between the detected response of an NM to an SNE and the characteristics of neutrons injected from the Sun towards the Earth during the event. It is shown that the total number of those solar neutrons with energy above $300 \mathrm{MeV}$ can be obtained directly from the timeintegrated NM response irrespectively of the neutron energy spectrum. We calculate the normalisation curve which allows one immediately to calculate the total number of the solar neutrons $(>300 \mathrm{MeV})$ from the monitor response to neutrons. The accuracy obtained for the number of neutrons is enough for the purpose of solar-flare studies and for testing different models.

Section 7 presents remarks on problems and perspectives of the study of solar neutrons by means of the World Neutron Monitor Network.

\section{Preliminary remarks}

One of the main difficulties encountered so far in the detection of high-energy solar neutrons by means of a neutron monitor is the weakness of the informative signal with respect to the background level $N_{b}$, which is caused by GCR. The mean value of $N_{b}$ depends on geographical coordinates and altitude of the NM location, and can be roughly described by the following approximate relationship:
$N_{b}=N_{o} \cdot r \cdot f\left(P_{c}\right) \cdot \exp \left(\frac{-\kappa-1033 g \cdot \mathrm{cm}^{-2}}{\lambda_{C R}}\right) \cdot \Delta t$,

where $N_{o} \approx 70$ counts/s is the average count-rate of a 6NM64 high-latitude sea-level NM (Debrunner, 1994); $f\left(P_{c}\right) \propto\left(1-\exp \left(-a P_{c}^{-k}\right)\right)$ is the function taking into account the latitude effect (see Stoker, 1994), where $P_{c}$ is the geomagnetic cut-off rigidity at the NM location; $\lambda_{C R} \approx 140 \mathrm{~g} / \mathrm{cm}^{2}$ is the average attenuation length of cosmic rays in the Earth's atmosphere; $\kappa$ is the atmospheric depth $\left(\mathrm{g} / \mathrm{cm}^{2}\right)$ at the NM location; $\Delta t$ is the data collection time; $r=n / 6$, where $n$ is the number of counters in a NM64-type monitor. For a comparison of count-rates of different types of NMs it is necessary to know the ratio $R$ of the integral efficiencies of the standard 6IGY and 6NM64 monitors, which depends slightly on time as well as on altitude and location of the NMs. For an IGY-type monitor, the expression for the factor $r$ in Eq. (1) is $r=n / 6 \cdot R$, where $n$ is the number of counters. In papers by Hatton and Carmichael (1964) and Hatton (1971) a value of $R=0.05$ is given for the high latitude and sea level, and this value is usually used when comparing count-rates of different monitors. On the other hand, low-latitude Jungfraujoch (Debrunner et al., 1987) and Haleakala (Pyle, 1993) mountain stations are equipped with counters of both types. The ratio of background count-rates of different types of monitors from these stations corresponds to the value of $R \approx 0.07$.

A dispersion $\sigma_{b}^{2}$ of the background is determined by assuming random, normally distributed fluctuations of the background count-rate. One can consider the dispersion in the form $\sigma_{b}^{2} \approx m N_{b}$, where the value of $m$ can be from 1.4 to 4 depending on the geomagnetic cut-off rigidity and the atmospheric depth at NM location. The value of $m$ corresponds to the multiplicity of registration of the secondary cascade nucleons (Iucci et al., 1984). In most cases, one can take $\sigma_{b}^{2} \approx 2 N_{b}$ (Belov et al., 1987).

In a general case, the mean error $\sigma$, of a NM countrate can be written in the form:

$\sigma^{2}=\sigma_{s}^{2}+\sigma_{b}^{2}+\zeta^{2}$,

where $\sigma_{s}^{2}$ is the dispersion of the signal, $\sigma_{b}^{2}$ is dispersion of the background, and $\zeta^{2}$ takes into account other sources of the error (instrumental errors, systematic errors, etc.). Usually, the duration of an SNE is from several minutes to several tens of minutes. One can consider the value of $N_{b}$ to be constant during such a period. However, in the case of a longer event it is necessary to take into account the possibility of a count-rate trend. The values of $\sigma_{s}$ and $\sigma_{b}$ are determined by assuming normally distributed random fluctuations of NM responses to solar neutrons and cosmic rays, respectively. Usually, $\sigma_{s}^{2} \ll \sigma_{b}^{2}$ because of the low signal level in comparison with the background. In most cases, one can assume that the value of $\zeta^{2}$ is negligible in comparison with $\sigma_{b}^{2}$. However, the value of $\zeta^{2}$ can be significant due to a subjective factor (e.g. the background level is erroneously calculated). For rough estimates, one can assume that $\sigma \cong \sigma_{b} \approx \sqrt{2 \cdot N_{b}}$. However, this expression is not accurate enough for detailed analysis. Besides, the value of $N_{b}$ varies in time 
depending on the phase of solar activity cycle, local time, barometric pressure, state of the interplanetary medium, etc. Thus, when analysing NM data, it is necessary to take the averaged count-rate immediately before the event as a background and calculate the value of $\sigma$ from the original count-rates. In addition, the trend in countrate should be taken into account.

\section{The dependence of the response of NM to solar neutrons on observational conditions}

The response of a given NM to solar neutrons, $N_{n}$ (counts), collected during the interval $\Delta t$ can be written in the form (e.g. Debrunner et al., 1989):

$N_{n}=\int_{t}^{t+\Delta t} \int_{E_{t h}}^{\infty} F_{n}\left(E, t^{\prime}\right) \cdot S_{n}(E) \cdot d E \cdot d t^{\prime}$,

where $F_{n}$ is the flux of solar neutrons at the Earth's orbit $\left(\mathrm{m}^{-2} \mathrm{sec}^{-1} \mathrm{MeV}^{-1}\right), E$ is the neutron energy, $S_{n}\left(\mathrm{~m}^{2}\right)$ is the sensitivity of the NM with respect to solar neutrons, $E_{t h}$ is the threshold energy $(\approx 50 \mathrm{MeV})$ for detection of solar neutrons by an NM.

The sensitivity of an NM to solar neutrons depends on the altitude of the NM location and the solar zenith angle. Using the following formula, one can determine the approximate value of the solar zenith angle, $\alpha$, for a given time and day of observation:

$$
\begin{aligned}
& \cos \alpha=\sin \varphi \cdot \sin \left(\frac{2 \pi}{365} t_{d}\right) \cdot \sin \varepsilon+\cos \varphi \\
& \sqrt{1-\sin ^{2}\left(\frac{2 \pi}{365} t_{d}\right) \sin ^{2} \varepsilon} \cdot \cos \left(\pi \cdot\left(\frac{T}{12}+\frac{\lambda}{180}-1\right)\right),
\end{aligned}
$$

where $\varphi$ and $\lambda$ are the latitude and the longitude of the NM location, respectively, $\varepsilon=23.5^{\circ}$ is the inclination of the equator relative to the ecliptic plane, $t_{d}$ is the number of days after the spring equinox, $T$ is the time $\mathrm{UT}$ in hours.

For a fixed flux of solar neutrons, the response of a given NM to solar neutrons is higher at a higher altitude of the NM and a smaller zenith angle. The northern hemisphere is more favourable for the neutron obser- vations during the northern summer season, while the southern hemisphere is good for northern winter observations.

In Sect. 5 the results of calculations of the sensitivity of an NM with respect to solar neutrons will be discussed. These calculations have been carried out by means of Monte Carlo simulations of the cascading of vertically $\left(\alpha=0^{\circ}\right)$ incident solar neutrons into the Earth's atmosphere. Debrunner et al. (1990) presented the results of the sensitivity calculation for the value of $\alpha$ up to $42.5^{\circ}$. However, the statistical error of those calculations for $\alpha>30^{\circ}$ is high. The problem of the angular dependence of the sensitivity is not yet clear. Therefore, an approximate empirical expression is usually used for the analysis of the response of the $\mathrm{NM}$ and for the comparison of responses of different NMs. The widely used expression is (e.g. Iucci et al., 1984; Takahashi et al., 1987)

$N_{n} \propto \exp \left(\frac{-\kappa}{\lambda_{n} \cdot \cos \alpha}\right)$

where $\lambda_{n} \approx 100 \mathrm{~g} / \mathrm{cm}^{2}$ is the attenuation length of solar neutrons in the Earth's atmosphere.

Though observational data of the well-studied SNE of 3 June 1982, obtained from several NMs with values of $\alpha$ up to $32.5^{\circ}$ (see Chupp et al., 1987), were in a good accordance with Eq. (5), Debrunner et al. (1990) noted that their Monte Carlo simulations of $S_{n}$ yielded lesspronounced angular dependence than that given by Eq. (5).

On 24 May 1990 the strongest SNE known so far (Shea et al., 1991) was detected (see Table 1). The event was detected with high significance by three neutron monitors (Climax, Calgary and Mexico) (the signal had an amplitude greater than 20 standard deviations of the background). Responses of another four stations to solar neutrons from the same event were higher than $2 \sigma$ and three monitors registered the event with low significance. The value of the solar zenith angle varied from $29^{\circ}$ to $65^{\circ}$ for different stations located at different altitudes from the sea level to $680 \mathrm{~g} / \mathrm{cm}^{2}$ (see Table 1). Debrunner et al. (1993) and Kovaltsov et al. (1993) noted that the observations of this event disagreed with Eq. (5), which can only be used when the Sun is near zenith i.e. for $\alpha \approx 0$. For large values of the solar zenith angle, the NM's sensitivity function is underestimated.

\begin{tabular}{|c|c|c|c|c|c|}
\hline neutron monitor & type & $\cos \alpha$ & $\kappa, \mathrm{g} / \mathrm{cm}^{2}$ & $N_{n}, \mathrm{cnts} / 5 \mathrm{~min}$ & $\sigma, \mathrm{cnts} / 5 \mathrm{~min}$ \\
\hline Mexico & $6 \mathrm{NM}$ & 0.8435 & 790 & 15214 & 404 \\
\hline Calgary & $12 \mathrm{NM}$ & 0.8269 & 895 & 10357 & 322 \\
\hline Inuvik & $18 \mathrm{NM}$ & 0.6712 & 1023 & 2539 & 366 \\
\hline Deep River & $48 \mathrm{NM}$ & 0.6137 & 1020 & 3872 & 456 \\
\hline Durham & $18 \mathrm{NM}$ & 0.5504 & 1029 & 508 & 261 \\
\hline Climax & $12 \mathrm{IGY}$ & 0.8642 & 680 & 6896 & 192 \\
\hline Mt.Washington & $12 \mathrm{IGY}$ & 0.5542 & 820 & 241 & 115 \\
\hline Newark & $9 \mathrm{NM}$ & 0.6056 & 1030 & 619 & 206 \\
\hline Goose Bay & $18 \mathrm{NM}$ & 0.4413 & 1027 & 269 & 375 \\
\hline Magadan & $18 \mathrm{NM}$ & 0.4227 & 988 & 276 & 330 \\
\hline
\end{tabular}

Table 1. Responses of neutron monitors to the solar-neutron event of 24 May 1990 during the interval 2050-2055 UT 
The underestimation is due to the fact that Eq. (5) takes into account secondary nucleons moving along the line of sight only. However, $\kappa \gg \lambda_{n}$ for all the monitors and the particles take part in several interactions before they reach an NM. Thus, the nucleons scattered into large angles play a significant role as well. Debrunner et al. (1993) showed that the observations of the 24 May 1990 SNE can be fitted by calculations of $S_{n}$ (Debrunner et al., 1990) within the limits of both observational and Monte Carlo statistical errors. Kovaltsov et al. (1993), on the basis of the data analysis, suggested a new approximate empirical expression which can be used for a wide range of the solar zenith angles $\alpha$ :

$N_{n} \propto \cos ^{m} \alpha \cdot \exp \left(\frac{-\kappa}{\lambda_{n}}\right)$.

On the basis of experimental data of the 24 May 1990 SNE, we tested the hypothesis that the angular dependence of NM response to solar neutrons can be described using Eq. (6). This was done by varying the atmospheric attenuation length $\lambda_{n}$ for neutrons and the exponent $m$ of $\cos \alpha$ for the angular dependence. The value of $R_{n}$, the ratio of integral efficiencies of standard 6IGY and 6NM64 monitors with respect to solar neutrons, was used as an additional parameter. The following two criteria were chosen: (i) the $\chi^{2}$ criterion at the significance level of $10 \%$ and (ii) the sign criterion (a model curve must lie between experimental points). For the study we made use of the data summarised in Table 1.

Figure 1 shows an area of possible values of the parameters in the $\lambda_{n}-m$ plane. The thin curve limits the area for the value of $R_{n}=0.07$, while the thick curve is a contour of the whole area of possible parameters $R_{n}=0.05-0.09$. The point corresponds to the best fit values of the parameters: $\lambda_{n}=104 \mathrm{~g} / \mathrm{cm}^{2}, m=4.0$, $R_{n}=0.07$. Figure 1 shows that the area of possible values is quite narrow. The value of the attenuation length $\lambda_{n}$ is found to be $100-110 \mathrm{~g} / \mathrm{cm}^{2}$. The value of $m$ appeared to be $3.8-4.5$ with the most probable value of 4.0. The ratio of the integral efficiencies $R_{n}$ of the two

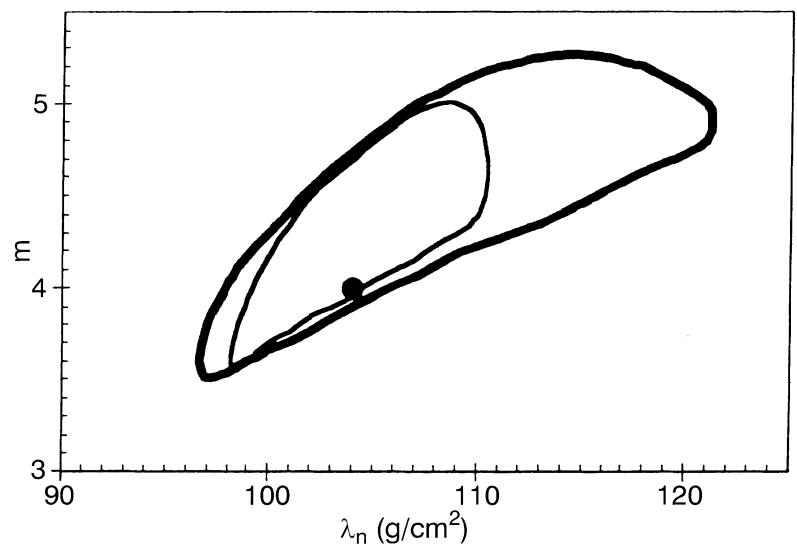

Fig. 1. Values of parameters $m$ and $\lambda_{n}$ of the empirical expression given by Eq. (6) for the neutron-monitor response to solar neutrons. The calculations were made for the 24 May 1990 solar-neutron event. The point corresponds to the best fit parameters types of standard monitors is $0.05-0.09$ with the most probable value of 0.07 .

Thus, we believe that the functional form of the most optimal sensitivity function of an NM to solar neutrons can be described by Eq. (6) with $\lambda_{n}=100 \mathrm{~g} / \mathrm{cm}^{2}, m=4.0$ and $R_{n}=0.07$. These values will be used throughout this paper.

Recently, Smart et al. (1995) suggested an analogy that the atmospheric nucleon cascade can be considered as a needle-type beam of solar neutrons which converges by about $3^{\circ}$ per $\lambda_{n}$ of passed air mass. Thus, the "path" of the particles becomes shorter in comparison with the line-of-sight path. Their results are in a good agreement with Eq. (6). Developing their approach, we considered the cascade as consisting of inelastic processes, described by the exponential decay of the flux intensity, and elastic processes leading to scattering of the nucleons. We simulated the process of a sequenced scattering of solar neutrons in the Earth's atmosphere, by means of the Monte Carlo technique. We did not take into account processes leading to changes of the primary particle's energy. This is a goal of our further research. It appears as if the approximation Eq. (6) agreed rather well with the simulation results in the whole range of atmospheric depth and solar zenith angle values.

The observational data of the well-known SNE of 3 June 1982 are in good agreement with Eq. (6) as well. Note that preliminary analysis of recently presented NM records of the 6 June 1991 SNE (Muraki et al., 1995) shows that the data agree with Eq. (6) while they contradict Eq. (5). Thus, at least for a practical use, Eq. (6) seems to be quite useful.

In the following the possibilities of the World Neutron Monitor Network to detect neutrons of solarflare origin will be considered. The list of NMs, which are suitable for SNE registration, is summarised in Table 2. This table shows the geographical coordinates and altitudes of NMs, UT time of local noon $T_{\text {noon }}$, the signal-noise ratio $N_{n} / \sigma$ (for an SNE on 3 June 1982 if the event had occurred at local noon. Observations of $\gamma$ radiation and neutrons of solar-flare origin carried out by both ground-based and space-borne detectors, during several recent cycles of solar activity, have shown that solar flares with such intensive high-energy neutron production as was observed on 3 June 1982, may be expected as frequently as several times per cycle (Mandzhavidze and Ramaty, 1992). Polar monitors are not included in Table 2 as they cannot reliably detect an SNE due to strong flux of GCR and large solar zenith angle in polar regions. Figure 2 shows the level at which the World Neutron Monitor Network could detect an event similar to the one on 3 June 1982 depending on the time of occurrence of the event. One axis corresponds to the date, the other to the time of day. Different shadings in Fig. 2 correspond to various values of the signal-noise ratio $N_{n} / \sigma$, where $N_{n}$ is the response of an NM to solar neutrons and $\sigma$ is the mean error of the NM count-rate. For Table 2 and Fig. 2, the value of $\sigma$ was estimated as $\sqrt{2 \cdot N_{b}}$, where $N_{b}$ was obtained from Eq. (1) for $\Delta t=5 \mathrm{~min}$. The values of $N_{n}$ for the NMs have been normalised with respect to the response of the Lomnický 
Table 2. The list of neutron monitors ordered according to their ability to detect solar neutrons

\begin{tabular}{|c|c|c|c|c|c|c|c|}
\hline Name & lat & long & $\begin{array}{l}P_{c} \\
G V\end{array}$ & $\begin{array}{l}\text { alt } \\
\mathrm{m}\end{array}$ & type & $\begin{array}{l}\mathrm{T}_{\text {noon }} \\
\mathrm{UT}\end{array}$ & $\begin{array}{l}N_{n} / \sigma \\
\max \end{array}$ \\
\hline Haleakala1 & 20.72 & 203.72 & 12.91 & 3030 & $18 \mathrm{NM}$ & 22.419 & 37.7 \\
\hline Alma-AtaB & 43.25 & 76.92 & 6.61 & 3340 & $18 \mathrm{NM}$ & 6.872 & 32.7 \\
\hline Mt-Norikura & 36.11 & 137.55 & 11.48 & 2770 & $12 \mathrm{NM}$ & 2.830 & 23.8 \\
\hline Jungfraujoch1 & 46.55 & 7.98 & 4.61 & 3475 & $3 \mathrm{NM}$ & 11.468 & 13.1 \\
\hline Mexico & 19.33 & 260.82 & 8.60 & 2274 & $6 \mathrm{NM}$ & 18.612 & 12.7 \\
\hline Lomnický Štit & 49.20 & 20.22 & 3.98 & 2634 & $8 \mathrm{NM}$ & 10.652 & 11.0 \\
\hline Tsumeb & -19.20 & 17.58 & 9.21 & 1240 & $18 \mathrm{NM}$ & 10.828 & 10.7 \\
\hline Huancayo & -12.03 & 284.67 & 12.92 & 3400 & $12 \mathrm{IGY}$ & 17.022 & 8.8 \\
\hline Jungfraujoch2 & 46.55 & 7.98 & 4.61 & 3475 & $18 \mathrm{IGY}$ & 11.468 & 8.3 \\
\hline Haleakala2 & 20.72 & 203.72 & 12.91 & 3030 & $12 \mathrm{IGY}$ & 22.419 & 8.0 \\
\hline Samarkand & 39.60 & 66.9 & 7.50 & 830 & $24 \mathrm{NM}$ & 7.540 & 7.3 \\
\hline Climax & 39.37 & 253.82 & 2.99 & 3400 & $12 \mathrm{IGY}$ & 19.079 & 6.6 \\
\hline Tokyo & 35.75 & 139.72 & 11.63 & 20 & $28 \mathrm{NM}$ & 2.685 & 5.6 \\
\hline Tashkent & 41.33 & 69.62 & 7.50 & 565 & $18 \mathrm{NM}$ & 7.359 & 5.4 \\
\hline Deep River & 46.10 & 282.5 & 1.14 & 145 & $48 \mathrm{NM}$ & 17.167 & 5.1 \\
\hline Tbilisi & 41.72 & 44.8 & 6.73 & 510 & $18 \mathrm{NM}$ & 9.013 & 4.9 \\
\hline Morioka & 39.70 & 141.13 & 10.23 & 131 & $18 \mathrm{NM}$ & 2.591 & 4.5 \\
\hline Calgary & 51.08 & 245.87 & 1.08 & 1128 & $12 \mathrm{NM}$ & 19.609 & 4.2 \\
\hline Darwin & -12.42 & 130.87 & 14.09 & 0 & $9 \mathrm{NM}$ & 3.275 & 3.7 \\
\hline Alma-AtaA & 43.25 & 76.92 & 6.61 & 775 & $6 \mathrm{NM}$ & 6.872 & 3.5 \\
\hline Rome & 41.90 & 12.52 & 6.32 & 60 & $17 \mathrm{NM}$ & 11.165 & 3.5 \\
\hline Irkutsk & 52.47 & 104.03 & 3.64 & 500 & $18 \mathrm{NM}$ & 5.065 & 3.2 \\
\hline Hermanus & -34.42 & 19.22 & 4.58 & 26 & $12 \mathrm{NM}$ & 10.719 & 3.1 \\
\hline Brisbane & -27.50 & 153.01 & 6.99 & 2 & $9 \mathrm{NM}$ & 1.799 & 3.1 \\
\hline Durham & 43.10 & 289.17 & 1.58 & 3 & $18 \mathrm{NM}$ & 16.722 & 3.0 \\
\hline Mt-Wellington & -42.92 & 147.23 & 1.80 & 725 & $6 \mathrm{NM}$ & 2.185 & 3.0 \\
\hline Kiev & 50.72 & 30.30 & 3.57 & 120 & $18 \mathrm{NM}$ & 9.980 & 2.8 \\
\hline Potchefstroom & -26.68 & 27.1 & 7.00 & 1351 & $15 \mathrm{IGY}$ & 10.193 & 2.6 \\
\hline Kerguelen & -49.35 & 70.27 & 1.14 & 33 & $18 \mathrm{NM}$ & 7.315 & 2.6 \\
\hline Mt-Washington & 44.30 & 288.7 & 1.46 & 1909 & $12 \mathrm{IGY}$ & 16.753 & 2.4 \\
\hline Newark & 39.68 & 284.25 & 2.09 & 50 & $9 \mathrm{NM}$ & 17.050 & 2.4 \\
\hline Sverdlovsk & 56.73 & 61.07 & 2.23 & 300 & $18 \mathrm{NM}$ & 7.929 & 2.3 \\
\hline Moscow & 55.47 & 37.32 & 2.43 & 200 & $18 \mathrm{NM}$ & 9.512 & 2.3 \\
\hline Goose Bay & 53.27 & 299.6 & 0.64 & 46 & $18 \mathrm{NM}$ & 16.027 & 2.2 \\
\hline Kiel & 54.73 & 10.13 & 2.36 & 54 & $18 \mathrm{NM}$ & 11.325 & 2.2 \\
\hline Alma-AtaC & 43.25 & 76.92 & 6.61 & 1650 & $12 \mathrm{IGY}$ & 6.872 & 2.2 \\
\hline Hobart & -42.90 & 147.33 & 1.84 & 15 & 9NM & 2.178 & 2.2 \\
\hline Fukushima & 37.75 & 140.48 & 10.61 & 66 & $4 \mathrm{NM}$ & 2.635 & 2.1 \\
\hline Dourbes & 50.10 & 4.6 & 3.34 & 225 & 9NM & 11.693 & 2.1 \\
\hline Magadan & 60.12 & 151.02 & 2.09 & 220 & $18 \mathrm{NM}$ & 1.932 & 1.8 \\
\hline Apatity & 67.55 & 33.33 & 0.57 & 182 & $18 \mathrm{NM}$ & 9.778 & 1.2 \\
\hline Mirny & -66.55 & 93.02 & 0.03 & 38 & $18 \mathrm{NM}$ & 5.799 & 1.1 \\
\hline Terre-Adelie & -66.55 & 140.02 & 0.02 & 35 & $18 \mathrm{NM}$ & 2.665 & 1.1 \\
\hline Oulu & 65.02 & 25.5 & 0.78 & 15 & $9 \mathrm{NM}$ & 10.300 & 1.0 \\
\hline Inuvik & 68.35 & 226.28 & 0.17 & 21 & $18 \mathrm{NM}$ & 20.915 & 1.0 \\
\hline TixiBay & 71.58 & 128.92 & 0.48 & 0 & $18 \mathrm{NM}$ & 3.405 & 0.8 \\
\hline Mawson & -67.60 & 62.88 & 0.20 & 30 & $6 \mathrm{NM}$ & 7.808 & 0.6 \\
\hline
\end{tabular}

Stit (8NM64) to solar neutrons during 11:45-11:50 UT of 3 June 1982. Note that though the values of $\sigma$ and $N_{n}$ are approximate estimates, they can be used to study the general properties of the network. "Maps" $a$ and $b$ of Fig. 2 are plotted according to Eqs. (5) and (6), respectively. The value of $N_{n} / \sigma$ corresponds to the most sensitive monitor, at the time of the SNE. One can see that the sensitivity of the world network to solar neutrons is much higher than earlier expected. The network can be used for continuous routine observations of SNEs, which is an advantage in comparison with space-borne experiments. The network can detect such strong events as the one on 3 June 1982 at a level $3 \sigma$ or better, during $93 \%$ of observation time, events threetimes weaker $54 \%$, and events weaker by an order of magnitude for $9 \%$ of the observation time. The most sensitive to solar neutrons are the high-altitude monitors located at low and middle latitudes: Haleakala, AlmaAta, Mt. Norikura, Jungfraujoch, Mexico, Lomnický Štit and Tsumeb (see Table 2). The network has a "dead time" with respect to solar neutrons (i.e. the network cannot detect even rather strong SNE) during morning hours, 04-08 UT, in winter time ("white area" in Fig. 2b). The "dead time" is caused by the natural absence of large mountain detectors in the region of Indian Ocean and Australia. 

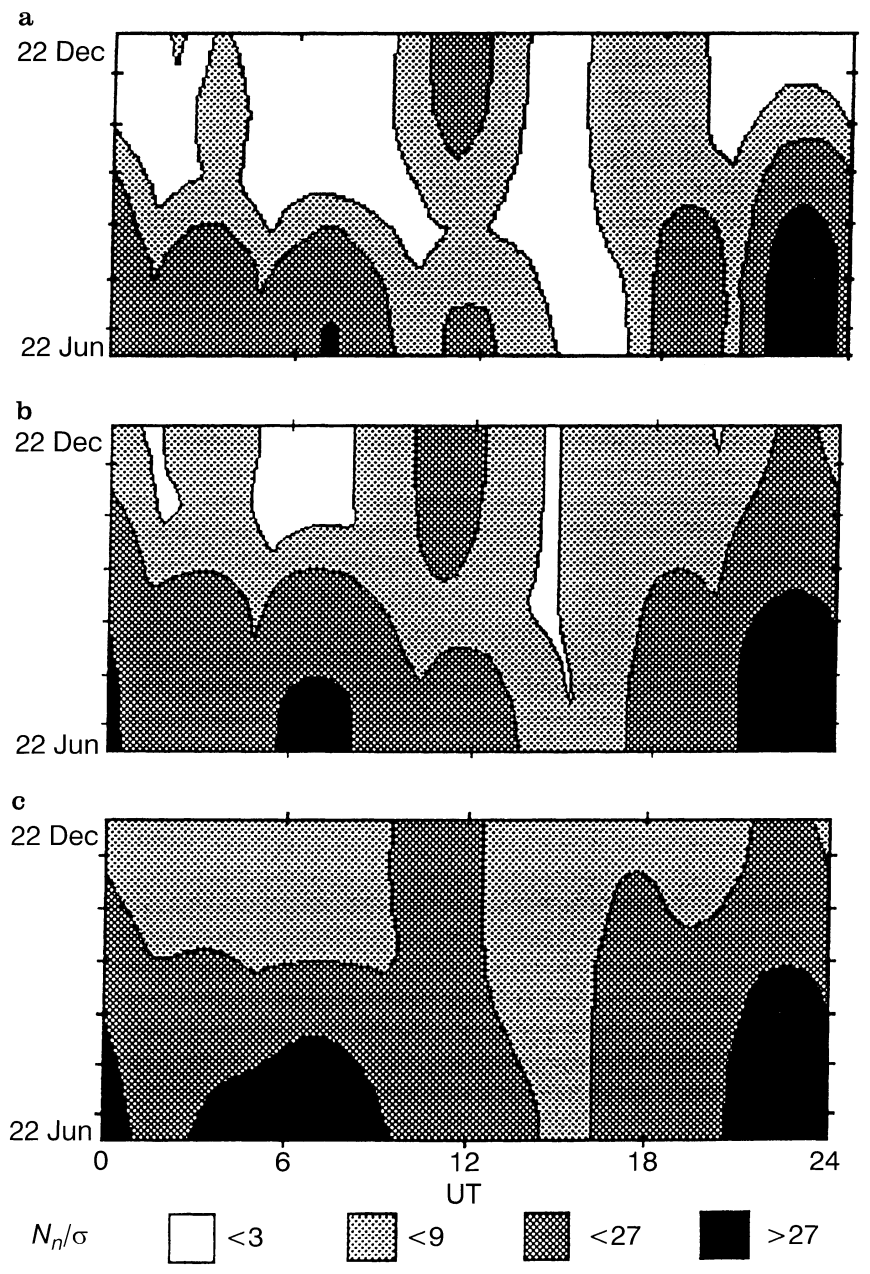

Fig. 2a-c. The sensitivity of the World Neutron Monitor Network to an intense solar-neutron event comparable to that of 3 June 1982 as a function of time of occurrence of the event. The shadowing corresponds to the significance of the detection of the event, in terms of $N_{n} / \sigma$. a The network sensitivity according to Eq. (5); b The network sensitivity according to Eq. (6); c The network sensitivity when the weighted summation of responses is applied

\section{Weighted summation of NM responses}

Assuming that responses of several NMs on the dayside of the globe are caused by a single SNE, one can use the information of the SNE not only from the NM with the largest response but from all the responding stations. The combined processing of different NM responses can be carried out using the techniques applied to a series of data with different values of accuracy (e.g. Hudson, 1964).

Monitors detect secondary nucleon flux initiated by solar neutrons in the Earth's atmosphere. The value of the flux depends on altitude and solar zenith angle of the observer [see Eq. (6)]. In order to consider responses of different NMs which have detected the event as independent measurements of the same flux of solar neutrons, one should normalise them to some standard conditions. Efimov et al. (1993) have suggested a 6NM64 monitor located at an altitude of $\kappa_{\mathrm{sc}}=$
$660 \mathrm{~g} / \mathrm{cm}^{2}$ as a standard instrument and standard observational conditions (this altitude corresponds to most mountain stations, Jungfraujoch, Alma-Ata, etc.) when the Sun is in the zenith, $\alpha_{\mathrm{sc}}=0^{\circ}$.

Let the value of $N_{n i}$ counts mean the response of the $i^{\text {th }} \mathrm{NM}$ to solar neutrons [see Eq. (5)]. This monitor is assumed to be located at an altitude corresponding to $\kappa_{i}\left(\mathrm{~g} / \mathrm{cm}^{2}\right)$ with a viewing of the Sun at the zenith angle $\alpha_{i}$. The signal-noise ratio for this NM is $N_{n i} / \sigma_{i}$, where $\sigma_{i}$ is the mean error of the background intensity. According to Eq. (6), the corresponding response $X_{i}$, for standard observational conditions should be:

$X_{i}=N_{n i} \cdot r_{n i} \cdot \exp \left(\frac{\kappa_{i}-\kappa_{s c}}{100 \mathrm{~g} / \mathrm{cm}^{2}}\right) \cdot \cos ^{-4} \alpha_{i}$,

where $r_{n i}=n_{i} / 6$ for NM64-type or $r_{n i}=R_{n} \cdot n_{i} / 6$ for IGY-type monitor with $n_{i}$ counters. One can see that the reduction of the response to the standard conditions is simply a scaling of the signal.

In order to obtain the weighted response of the NM network to an SNE one should make use of weight factors which account for the reliability of every separate measurement. As the weight of the $i^{\text {th }}$ measurement, the value $p_{i}=1 / \sigma_{i}^{* 2}$ is used, where $\sigma_{i}^{*}$ is the corresponding mean error of the value $X_{i}$. As long as the signal-noise ratio is constant at the scaling of the signal, one can obtain $\sigma_{i}^{*}=\sigma_{i} \cdot\left(X_{i} / N_{n i}\right)$.

For the standard conditions, the weighted response of all the considered monitors to the SNE can be written as:

$\xi=\frac{1}{p} \cdot \sum_{i} p_{i} X_{i}$

where $p=\sum p_{i}$ is the weight of the weighted response. The expected mean error of $\xi$, before the equalisation, is $\sigma_{\xi}=1 / \sqrt{p}$.

On the other hand, one can calculate the actual mean error of $\xi$, after the equalisation, as

$\sigma_{\xi}^{*}=\sqrt{\frac{1}{(n-1) p} \cdot \sum_{i=1}^{n} p_{i}\left(X_{i}-\xi\right)^{2}}$.

In a general case, $\sigma_{\xi}^{*} \neq \sigma_{\xi}$. They are random values as they depend on measurement errors of separate monitors which are random. If those measurements did not contain a systematic error, the expected values of $\sigma_{\xi}^{*}$ and $\sigma_{\xi}$ would be equal. In the present case, the systematic errors could be introduced through several sources such as (a) the response of NM not to solar neutrons but to protons, (b) the uncertainty of the reduction Eq. (7) and (c) instrumental systematic errors. If $\sigma_{\xi}^{*}<\sigma_{\xi}$, the origin of the difference is random, and one can take the value of

$\sigma_{\xi}^{f}=\left(\sigma_{\xi}^{*}+\sigma_{\xi}\right) / 2$

as the final estimate of the mean error of the value of $\xi$. On the other hand, if $\sigma_{\xi}^{*}>\sigma_{\xi}$, one should calculate the value of a factor $k$ : 
$k=\frac{\sigma_{\xi}^{* 2}-\sigma_{\xi}^{2}}{\sigma_{\xi}^{2}} \cdot \sqrt{\frac{n-1}{2}}$.

When $k<2$, then, most likely, the difference between $\sigma_{\xi}^{*}$ and $\sigma_{\xi}$ is of random origin, and one can write the final estimate of the mean error of $\xi$ as

$\sigma_{\xi}^{f}=\sigma_{\xi}^{*}$

For $k>2$, a systematic error exists in the series $X_{i}$. The factor $k$ allows a verification of the accuracy of Eq. (6) as well as of the time of onset of a GLE in the case of the GLE following the SNE.

Thus, the weighted responses of different NMs with respect to an NM under standard observational conditions (as defined earlier) can be presented in the form:

$N_{S C}=\xi \pm \sigma_{\xi}^{f}$,

where $\xi$ is determined by Eq. (8) and $\sigma_{\xi}^{f}$ can be calculated from Eqs. (11) or (13).

In the following, the weighted summation of $\mathrm{NM}$ responses is applied to the event of 3 June 1982 which was detected by six neutron monitors: Jungfraujoch, Lomnický Stit, Rome, Kiel (Chupp et al., 1987), Tsumeb (Stoker, 1987) and Alma-Ata (Zusmanovich and Shwartsman, 1987). The monitor most sensitive to solar neutrons at the flare time was the high-mountain monitor Lomnický Stit. It recorded the SNE at the maximum level of $N_{n} / \sigma=7.3$ during the interval 11:4511:50 UT. Figure 3a shows the time-profiles of countrates for the monitors. The count-rate is normalised to that for standard observational conditions. The timeprofile of weighted response for the six monitors is shown in Fig. 3b. The values of the weighted response $\xi$, reduced to the standard conditions, the mean error of $\xi, \sigma_{\xi}^{f}$, signal-noise ratio $\xi / \sigma_{\xi}^{f}$ for this event are listed in Table 3. For the maximum of the event the value of the signal-noise ratio for the weighted response is 12.1, which is significantly higher than that for any separate monitor. Thus, the summation of responses offers an improvement in the detection efficiency for solar neutrons. Note that in the case considered, the value of the $k$ factor was $<2$, demonstrating that the difference in the responses of separate NMs was of random origin. The advantages of the method become even more obvious if, for the event, the responses of the most sensitive monitors, Lomnický Štit and Jungfraujoch were not taken into account. The individual responses of the other four NMs did not exceed the level of $2.5 \sigma$. This value is too low for a reliable detection of the SNE. Fig. $3 \mathrm{c}$ shows the weighted response for the four monitors, excluding Lomnický Štit and Jungfraujoch.
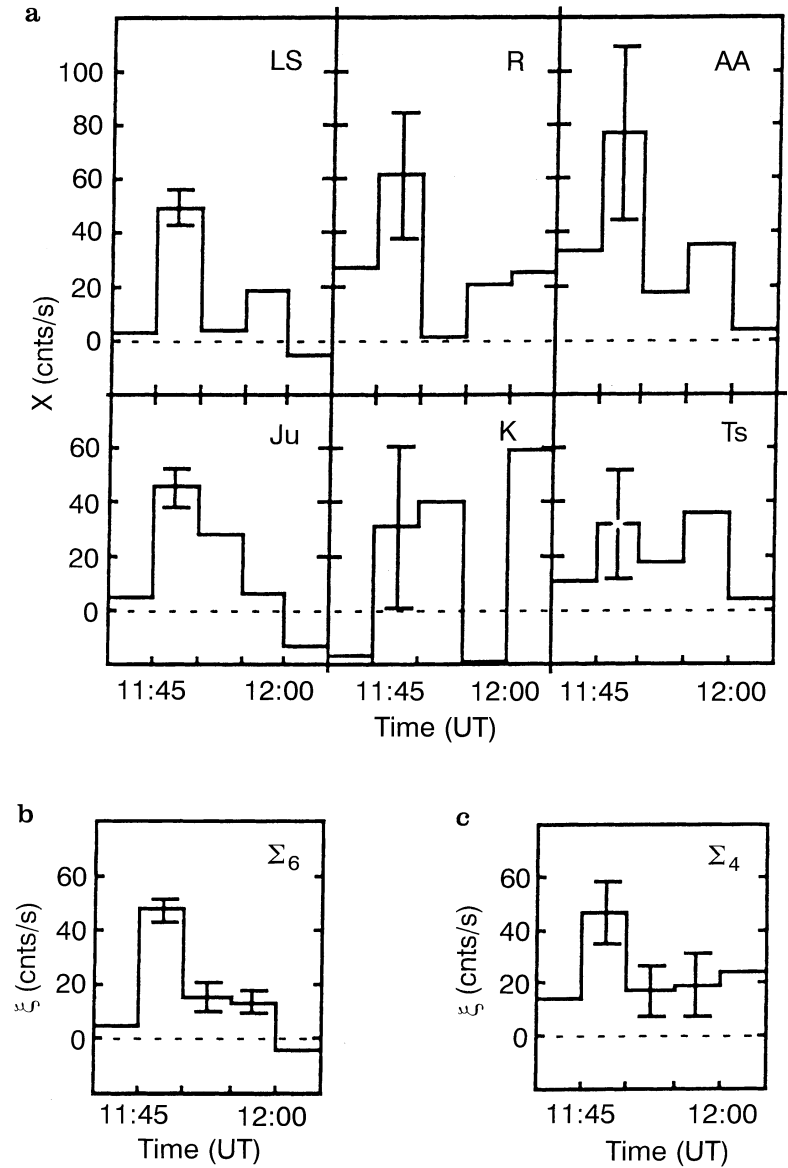

Fig. 3. a Responses of neutron monitors for the solar-neutron event on 3 June 1982 reduced to the standard observational conditions (see text): LS - Lomnický Štit; R - Rome; AA - Alma-Ata; Ju Jungfraujoch; K - Kiel; Ts - Tsumeb; b the weighted summed response $\sum_{6}$ of the six NMs; $\mathbf{c}$ the weighted summed response $\sum_{4}$ of four NMs (R, AA, K, Ts)

The signal-noise ratio for the weighted response is 5.1 for the 11:45-11:50 UT interval, and the SNE can be detected with a high significance.

The sensitivity of the World Neutron Monitor Network to solar neutrons, when the responses of separate NMs are weightedly summed, is shown in Fig. 2c. One can see that within the entire year the existing network can detect, with a significance of $\geq 3 \sigma$, an SNE as intense as that of 3 June, 1982. Events three times weaker can be reliably detected during $63 \%$ of the total observational time, and events ten times weaker during $15 \%$ of the time (compare with Fig. 2b). Thus, the use of weighted summation of NM responses significantly improves the efficiency of the network to detect weak SNEs.
Table 3. The weighted response $(\xi)$ of the World Neutron Monitor Network (counts/s) under the standard observational conditions to the solar neutron event of 3 June 1982; $\sigma_{\xi}^{f}=$ the mean error of $\xi$

\begin{tabular}{llllll}
\hline & & \multicolumn{3}{c}{ Time, UT } & \\
& $11: 40-11: 45$ & $11: 45-11: 50$ & $11: 50-11: 55$ & $11: 55-12: 00$ & $12: 00-12: 05$ \\
\hline$\xi$ & 5.1 & 47.3 & 15.1 & 13.4 & -4.5 \\
$\sigma_{\xi}^{f}$ & 3.9 & 3.9 & 5.4 & 4.4 & 6.2 \\
$\xi / \sigma_{\xi}^{f}$ & 1.3 & 12.1 & 2.8 & 3.05 & -0.7 \\
\hline
\end{tabular}




\section{Test of the NM sensitivity to solar neutrons}

In order to reconstruct the characteristics of the neutron injection from the Sun one should know the sensitivity of a monitor to solar neutrons as a function of their energy. There exist several calculations of the sensitivity of NM for solar neutrons, $S_{n}$, (Debrunner et al., 1983, 1989, 1990; Chupp et al., 1987; Efimov and Terekhov, 1988; Gueglenko et al., 1990a; Shibata 1994). The results of the calculations differ significantly from each other. For instance, Fig. 4 shows the results of calculations of monitor sensitivity for the actual observational conditions of the 3 June 1982 SNE carried out by Debrunner et al. (1983, 1989, 1990), Chupp et al., (1987) and Shibata (1994) for Jungfraujoch (18IGY) and Lomnický Štit (8NM64) monitors, and Efimov and Terekhov for Lomnický Štit NM (Efimov and Terekhov, 1988; Gueglenko et al., 1990a). Hereafter, we denote the corresponding sensitivities as IGY-D and IGY-Sh as well as NM64-D, NM64-Sh and NM64-E, respectively. It is seen from Fig. 4 that in the energy range below 300 $\mathrm{MeV}$ there is great difference between the normalised values as well as between shapes of the sensitivity $S_{n}$ (e.g. Shibata, 1994).

It is well known that the integral efficiency of the IGY monitor for solar neutrons is an order of magnitude lower than that of the NM64 monitor (Hatton, 1971). Thus, the ratio of these efficiencies can serve as a first rough criterion for testing the sensitivities. The sensitivity of the NM64-D is much lower than the sensitivities of both NM64-Sh and NM64-E, and also lower than the sensitivity for the IGY-D. On the other hand, the ratio between the NM64-E and the IGY-D sensitivities is reasonable and in accordance with the ratio $R_{n}$ of integral efficiencies of the 6IGY and 6NM64 monitors, under the same observational conditions, for solar neutrons, observed on 24 May 1990 (see Sect. 3). Taking into account that the sensitivities given by

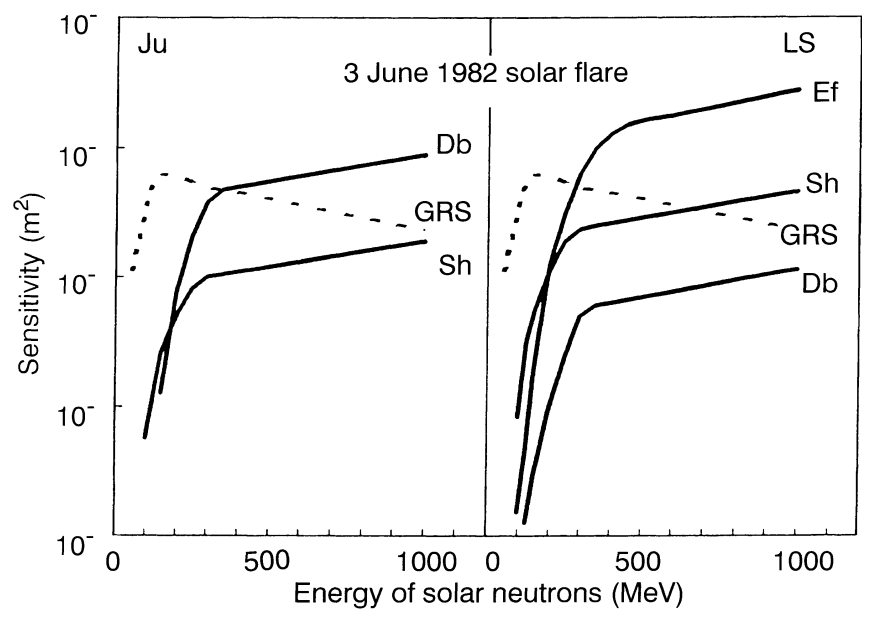

Fig. 4. The sensitivities of Jungfraujoch 18 IGY (left plot) and Lomnický Śtit 8NM64 (right plot) neutron monitors to solar neutrons for the 3 June 1982 solar-neutron event. The sensitivities are: Db IGY-D and NM64-D; Sh - IGY-Sh and NM64-Sh; Ef - NM64-E. The sensitivity of the GRS/SMM detector to neutrons is indicated with a dotted line
Debrunner et al. (IGY-D and NM64-D) were based on the same atmospheric nucleon cascade simulation, we believe that the value of the integral efficiency of NM64(Debrunner et al., 1989, 1990) type monitor was underestimated by a factor of $\approx 20$. Thus, we exclude the NM64- D sensitivity from further consideration. The IGY-D sensitivity seems to be correct. Both IGY-Sh and NM64-Sh sensitivities are in accordance with each other, although they are 4-6 times smaller than those by other groups (IGY-D and NM64-E). Thus, a need for more reliable tests for comparison of NM sensitivities is apparent. A solar flare, for which information about neutron production is obtained not only from NMs, but also from the detection of direct solar neutrons, neutron-decay protons, and $\gamma$-emission aboard satellites, can serve as such a test.

The SNE of 3 June 1982 was selected for this test. Neutrons were reliably detected by GRS aboard the SMM satellite, Lomnický Štit (8NM64 at $2632 \mathrm{~m}$ altitude, solar zenith angle $29^{\circ}$ ) and Jungfraujoch (18IGY, $3554 \mathrm{~m}, 24.5^{\circ}$ ) neutron monitors (Chupp et al., 1987). Protons from neutron decays in the interplanetary space were detected as well (Evenson et al., 1983). The flare has also been studied as a source of $\gamma$-ray emission (Ramaty and Murphy, 1987; Gueglenko et al., 1990b; Mandzhavidze and Ramaty, 1992). The observed ratios between the integral (over the entire SNE) responses (counts) of NMs and GRS/SMM were $R_{J u}=0.36 \pm 0.06$ for Jungfraujoch/GRS and $R_{L S}=0.61 \pm 0.09$ for Lomnický Stit/GRS. The GRS/SMM detector is sensitive to solar neutrons (Chupp et al., 1987) of lower energy than the neutrons observed by monitors (see Fig. 4). Therefore, the values of $R_{J u}$ and $R_{L S}$ depend on the steepness of the solar-neutron spectrum. Based on this fact and using the IGY-D sensitivity, Chupp et al. (1987) calculated for the event on 3 June 1982 the ratio $R_{J u}$, between the integral count rates of the GRS/SMM and the Jungfraujoch IGY monitor, and reconstructed the spectrum of injected solar neutrons. In this paper a similar approach was applied.

Following the approach of Chupp et al. (1987), the calculations of the ratios were made using a power-law spectrum $f(E) \propto E^{-G}$ for the injected neutrons with cutoff at $E_{\text {cut }}=2 \mathrm{GeV}$ and varying values of the spectral index, $G$. Developing their approach, we consider both $R_{J u}$ and $R_{L S}$, thus treating the case in two dimensions. Figure 5 shows the calculated values of $R_{J u}$ and $R_{L S}$ as well as observed values of $R_{J u}$ and $R_{L S}$ with $\pm \sigma$ and $\pm 2 \sigma$ uncertainty ellipses. The monitor sensitivities IGY-D (Fig. 5a) and IGY-Sh (Fig. 5b) were chosen for the Jungfraujoch monitor and NM64-E (Fig. 5a) and NM64-Sh (Fig. 5b) for the Lomnický Stit monitor. It is seen from the figure that IGY-D and NM64-E sensitivities yield the values $2.5-2.7$ for the spectral index $G$. This is in good agreement with the results obtained earlier by Chupp et al. (1987) using only the Jungfraujoch NM data. The spectrum of the injected neutrons must be much harder $(G=1.3-1.5)$ if the sensitivities IGY-Sh and NM64-Sh are used. Neither of the calculated curves crosses the $\pm \sigma$ area while both lines cross the $\pm 2 \sigma$ area. This could result from either a 


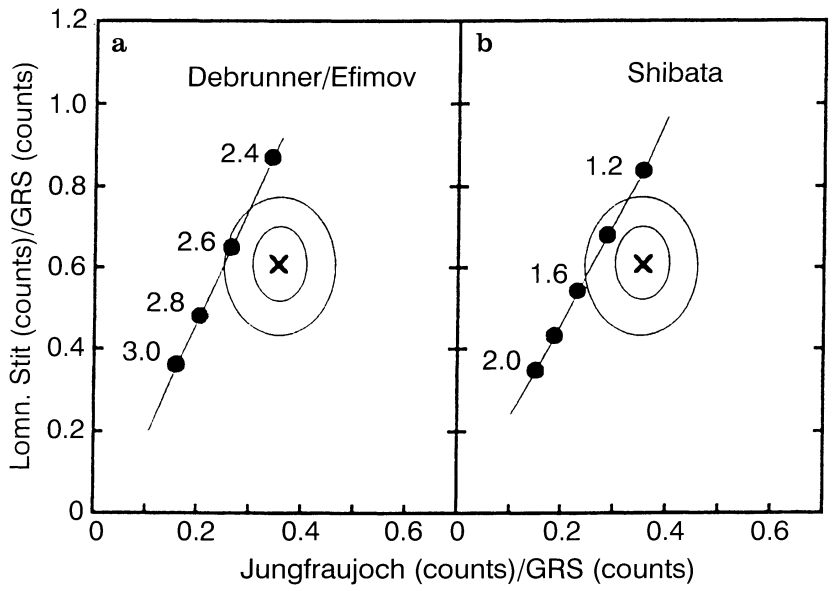

Fig. 5a, b. The ratio of the responses of neutron monitors to the response of the GRS instrument onboard the SMM spacecraft for the 3 June 1982 solar flare. The observed values are shown as crosses with $1 \sigma$ and $2 \sigma$ uncertainty ellipses. Lines correspond to calculated values of the ratio according to the IGY-D/NM64-E and IGY-Sh/NM64-Sh sensitivities (a and b plots, respectively). The figures correspond to values of the $G$ index of a power-law spectrum of solar neutrons

statistical artefact or a systematic error. The fact that a and $\mathrm{b}$ of Fig. 5 are similar speaks in favour of a systematic error. For instance, such an effect may arise if the ratio of the integral efficiencies of the two monitor types, $R_{n}$, is overestimated by $20-30 \%$. Shibata (1994) emphasised the importance of detailed knowledge of the sensitivity at a neutron energy $<300 \mathrm{MeV}$. However, even a considerable change in the shape of the function in this range could result in only slight changes of the spectral index $G$. The large difference in the values of $G$ mentioned is mainly due to different normalisations of the sensitivity $S_{n}(E)$ by different groups, but not due to different shapes of the sensitivities in the energy range below $300 \mathrm{MeV}$.

Figure 6 shows the calculated neutron spectra for the 3 June 1982 SNE using the most probable parameters for the neutron injection according to sensitivities given by IGY-D/NM64-E and IGY-Sh/NM64-Sh. Also plotted are the characteristics of neutrons injected from the Sun on 3 June 1982 as they were associated with $\gamma$-ray emission and proton fluxes from neutron decay of the same flare (Evenson et al., 1983; Ramaty and Murphy, 1987; Mandzhavidze and Ramaty, 1992). Also shown is neutron injection from the Sun corresponding to the observed flux of neutron-decay protons detected by ISEE-3 as well as neutrons corresponding to the observations of $\gamma$-emission in the $2.2-\mathrm{MeV}$-neutron capture line $n(p, d) \gamma$ and $\gamma$-emission from decay of high-energy $\pi^{\circ}$. Since pions and neutrons are produced simultaneously in solar flares, the generation rate of $\pi^{\circ}$ decay $\gamma$-rays $(>10 \mathrm{MeV})$ is proportional to the generation rate of neutrons $(>300 \mathrm{MeV})$. Estimates of the neutron spectrum were made using modern models of the 3 June 1982 flare (Gueglenko et al., 1990a; Mandzhavidze and Ramaty, 1992).

One can see from Fig. 6 that the injected neutron spectra obtained in the case of IGY-D/NM64-E sensitivities are in a good agreement with other observations.

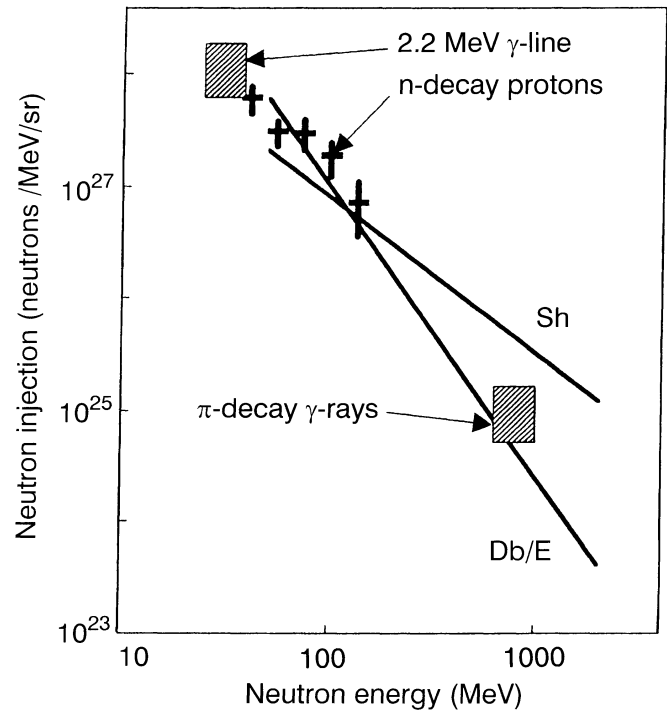

Fig. 6. Spectra of newly injected solar neutrons for the 3 June 1982 solar flare obtained using the IGY-D/ NM64-E (Db/ E) and IGY-Sh/ NM64-Sh (Sh) sensitivities in a comparison with neutron fluxes corresponding to observations of neutron-decay protons together with 2.2-MeV $\gamma$-line emission and pion decay $\gamma$-emission

The total number of neutrons with energy above 300 $\mathrm{MeV}$ injected from the Sun towards the Earth, as estimated from observations of $\pi$-decay $\gamma$-rays, was less than $2.5 \times 10^{28} \mathrm{sr}^{-1}$ for the flare. IGY-Sh/NM64-Sh sensitivities yield too high a flux of injected neutrons and too hard an energy spectrum. Based on observational data of the $2.2-\mathrm{MeV} \gamma$-line and neutron-decay protons, a steepening of this spectrum is expected below $100 \mathrm{MeV}$, which contradicts the established interpretation of the flare on 3 June 1982.

Recently, based on Climax (12IGY) NM data as well as on other observations of the flare emission, a reconstruction was made of the characteristics of solar-neutron injection from the Sun for the flare of 24 May 1990 (Kocharov et al., 1994; Kovaltsov et al., 1995a, b). The IGY-D sensitivity for the monitor was used for the reconstruction of the neutron spectrum. The flux of neutron-decay protons near the Earth was calculated and compared with high-energy proton observations by the GOES satellite (Kocharov et al., 1995). The calculated results showed a reasonable agreement with the precursor recorded by GOES. If the IGY-Sh sensitivity were used, the calculated flux of neutrondecay protons would be several times higher than the one observed.

Thus, we may conclude that both the IGY-Sh and NM64-Sh sensitivity functions of an NM to solar neutrons are most likely underestimated. The IGY-D and NM64-E sensitivities are acceptable. Note that this analysis refers mainly to the test of integral normalisations of calculations of the sensitivity of an NM with respect to solar neutrons carried out by different groups. The difference in the shape of sensitivity in the range of neutron energy below $300 \mathrm{MeV}$ is not crucial because, as will be shown in Sect. 6, the main contribution to the NM response, for not a very steep neutron spectrum, is 
due to neutrons with energy above $300 \mathrm{MeV}$. Though the shape of $S_{n}$ for high neutron energies as presented by different groups is almost the same and we have tested the integral normalisation, the question of the shape of $S_{n}$ in the range below $300 \mathrm{MeV}$ seems still to be open. More detailed simulations of the atmospheric nucleon cascade and NM sensitivity for various angles of solar neutrons are necessary for further research of SNEs by means of the World Neutron Monitor Network.

\section{Deduction of the number of solar neutrons from NM response}

To study processes in the area of neutron production during a solar flare, it is very useful to determine both spectral and temporal characteristics of the injection of high-energy neutrons from the Sun. Unfortunately, an NM measures an integral flux of particles in cascades, initiated by different neutrons, which are mixed in the atmosphere, and a separation of the responses becomes impossible. Therefore, when analysing the response of an NM to solar neutrons one has to make some a priori assumptions on the spectrum of neutrons and temporal behaviour of their injection. Assumptions are commonly made based on observations of other types of flareassociated high-energy radiation. A comparison of the calculated response of an NM with the actual response allows one to obtain possible values of the parameters of an a priori assumption model of the neutron injection. For instance, such an approach has been used by Chupp et al. (1987) for the analysis of the 3 June 1982 SNE as well as by Debrunner et al. (1993) and Kocharov et al. (1994) for the analysis of the 24 May 1990 SNE. However, this approach can be applied only for strong neutron events for which the time-profile of the NM response can be obtained. For a weaker SNE only the response of an NM integrated over the entire event can be obtained. This time-integrated response is determined by the following expression:

$Q_{n}=\frac{1}{R_{a}^{2}} \int_{E_{t h}}^{\infty} f(E) \cdot S_{n}(E) \cdot \exp \left(-\frac{R_{a}}{\gamma \tau_{n} v}\right) \cdot d E$,

where $f(E)\left(\mathrm{sr}^{-1} \mathrm{MeV}^{-1}\right)$, is the spectrum of neutrons injected from the Sun towards the Earth, integrated over the entire injection time. The exponent describes neutron decay in the interplanetary medium. $\tau_{n}$ is neutron decay time in intrinsic frame of reference. $S_{n}$ and $E_{t h}$ are the sensitivity and the threshold energy of neutron detection of a NM with respect to solar neutrons. $E, \gamma$ and $v$ are energy, Lorentz-factor and velocity of a neutron, respectively. $R_{a}=1 \mathrm{AU}$. One can easily obtain Eq. (15) when e.g. integrating Eq. (4) of Debrunner et al. (1993) over the injection time.

The question may arise, What information about the flare can be obtained from this time-integrated response of NM? Since an NM measures an integral flux it seems natural to determine from the detected value of $Q_{n}$ the total number of neutrons with energy above $E_{\text {norm }}$ injected from the Sun towards the Earth, which is:
$A_{n}\left(>E_{\text {norm }}\right)=\int_{E_{\text {norm }}}^{\infty} f(E) d E, \mathrm{sr}^{-1}$.

In a general case, in order to estimate the value of $A_{n}$ on the basis of detected $Q_{n}$ one should know the form and parameters of in situ neutron time-integrated spectrum, $f(E)$, which is unknown. In such a case, a priori assumptions have to be used. When such an approach is used the value of $E_{\text {norm }}$ should not be chosen arbitrarily. The value of $Q_{n}$ is fixed as a "detected" value, while the corresponding value of $A_{n}$ depends on the choice of $E_{\text {norm }}$ and the unknown spectral form of $f(E)$. The fact that $f(E)$ is unknown can be included as a systematic error of determination of $A_{n}$ from $Q_{n}$. For an arbitrary value of $E_{n o r m}$ this error is so high that the determination of $A_{n}$ from $Q_{n}$ without additional assumptions is impossible. Thus, the value of $E_{\text {norm }}$ should be chosen so that the systematic error is minimised. The fact that such a value $E_{\text {norm }}^{*}$ of $E_{\text {norm }}$ exists means that the value of $Q_{n}$ can be directly associated with the value of $A_{n}\left(>E_{\text {norm }}^{*}\right)$ irrespectively of the shape of $f(E)$. Therefore, one can estimate with some accuracy the value of the total number of neutrons injected from the Sun without any a priori assumption of the shape of $f(E)$.

In our study we used for the energy spectrum of injected neutrons (see e.g. Chupp et al., 1987) an exponential spectrum $f(E) \propto \exp \left(-E / E_{o}\right)$ with $E_{o}$ as the characteristic energy of the spectrum as well as a power-law spectrum $f(E) \propto E^{-G}$ with cut-off at energy $E_{\text {cut }}$. We calculated the response $Q_{n}$, of a NM64-type NM integrated over the entire event, using the sensitivity $S_{n}(E)$ NM64-E according to Efimov and Terekhov (1988), for a fixed total number of injected neutrons. For illustration of the results of the calculations, an exponential spectrum of injected neutrons is chosen, though all the conclusions below are true for power-law spectra as well. Figure. 7 shows the calculated value of $Q_{n}$ vs. $E_{o}$ for various values of the normalisation energy, $E_{\text {norm }}$. One can see that for $E_{\text {norm }} \approx 300 \mathrm{MeV}$ the dependence

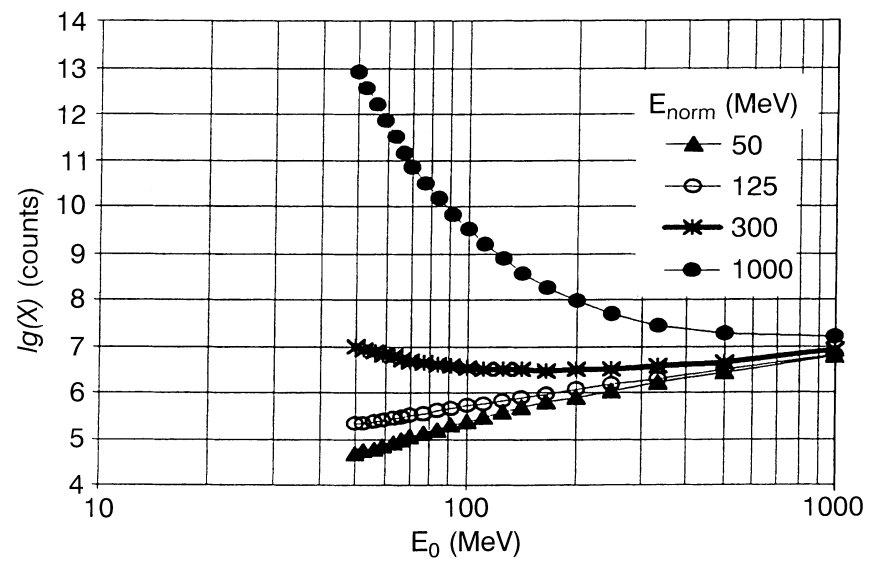

Fig. 7. The logarithmic response of a neutron monitor to solar neutrons for the standard observational conditions (see Sect. 4) as a function of the characteristic energy $E_{o}$ of an exponential solar-neutron spectrum. The total number of neutrons with energy above $E_{\text {norm }}$ injected from the Sun towards the Earth is fixed at $10^{30}$ neutrons/sr. Different curves correspond to different values of $E_{\text {norm }}$ 
of the response $Q_{n}$ on the neutron spectrum $f(E)$ is weak. In other words, the total number of neutrons with energy above $300 \mathrm{MeV}$ injected from the Sun towards the Earth can be deduced from the integrated response of a neutron monitor without knowledge of the shape of the neutron spectrum. The accuracy of deduction of the value of $A_{n}$ from $Q_{n}$, although not high (within a factor of 3), is enough to be used in study of high-energy processes taking place in solar flares. Note that the content of this section concerns SNEs for which a complex analysis of $\gamma$-emission and direct neutron detection is impossible. Otherwise, records of NM count-rates during an SNE can serve as an additional information for a comparison with other data and to check the validity of the models.

Using a normalisation energy of $300 \mathrm{MeV}$ the total number of high-energy solar neutrons injected from the Sun towards the Earth has been plotted against the atmospheric depth of NM location (Fig. 8). This figure shows (solid line) the number of neutrons injected from the Sun, $A_{n}(>300 \mathrm{MeV}) \mathrm{sr}^{-1}$, per count (above the background) of a 6NM64 monitor located at the altitude of $\kappa \mathrm{g} / \mathrm{cm}^{2}$, at a sub-solar point (solar zenith angle $\alpha=0^{\circ}$ ), collected during the entire SNE. Dotted lines correspond to estimated uncertainties. For the time-integrated response $Q_{n}$ of a certain NM located at the altitude of $\kappa \mathrm{g} / \mathrm{cm}^{2}$ with the solar zenith angle $\alpha$, the corresponding total number of neutrons $(>300 \mathrm{MeV})$ injected from the Sun towards the Earth can be calculated, using Eq. (6), as

$A_{n}(>300 \mathrm{MeV})=Q_{n} \cdot y(\kappa) \cdot \cos ^{-4} \alpha \cdot r_{n}, \mathrm{sr}^{-1}$,

where $y(\kappa)$ should be found from the curve in Fig. 8, $r_{n}=n / 6$ for NM64-type (or $r_{n}=R_{n} \cdot n / 6$ for IGY-type) monitor with $n$ counters. In the case of an SNE detected by several NMs, the weighted response $\xi$ of the network should be used for calculation of $A_{n}(>300 \mathrm{MeV})$ :

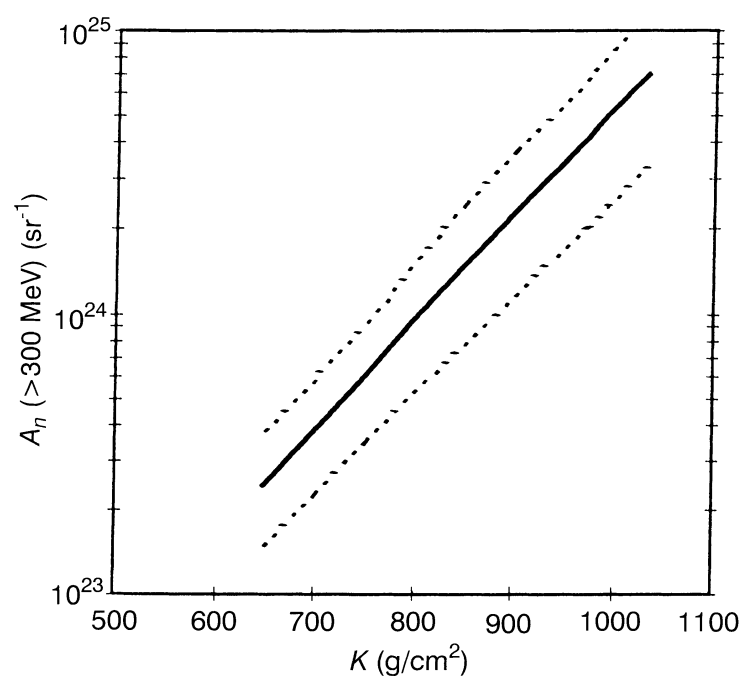

Fig. 8. The calculated number (solid line) of neutrons injected from the Sun towards the Earth per count of a 6NM64 monitor vs. the altitude of the NM station. The Sun is assumed to be in the zenith. Dotted lines correspond to estimated uncertainties of $\pm \sigma$
$A_{n}(>300 \mathrm{MeV})=\xi \cdot y(\kappa), \mathrm{sr}^{-1}$.

In order to illustrate how the approach works, let us consider the well-known case of the 3 June 1982 event. The weighted response of the Neutron Monitor Network, reduced to the standard observational conditions (a 6NM64 monitor located at the altitude of $\kappa=$ $660 \mathrm{~g} / \mathrm{cm}^{2}$ at sub-solar point, $\alpha=0^{\circ}$ ) was $\xi=24270$ counts above the background during the period 11:40 12:00 UT (see Table 3). According to Eq. (17a), the corresponding value of $A_{n}(>300 \mathrm{MeV})$ is $(4-10)$. $10^{27} \mathrm{sr}^{-1}$, which is in agreement with the value obtained by Chupp et al. (1987).

Thus, when using Fig. 8 and Eqs. (17), (17a), one can immediately estimate from the observed response of an NM to an SNE with an accuracy of a factor of 3, the total number of neutrons $(>300 \mathrm{MeV})$ injected from the Sun. Therefore, the approach can be recommended as a fast preliminary analysis of a strong SNE and for a regular study of a weaker SNE when additional information about injected neutrons cannot be obtained. This approach can also be applied to a solar flare for which only an upper limit of the NM response can be obtained from observations. From this upper limit response, the corresponding upper limit of the total number of injected neutrons can be estimated, which is also very important for solar-flare physics.

\section{Concluding remarks}

It has been shown that the ground-based World Neutron Monitor Network is a suitable tool for research of high-energy solar-flare neutrons. The advantages of this network are its continuos operation as monitor of solar and galactic particles, its relatively low cost of operation and its long continuity of observations (over several solar cycles). One of its main disadvantages is the fact that an NM detects not the original solar and galactic nucleons, but nucleons of an atmospheric cascade initiated by the primaries. This may introduce some errors in the study of solar neutrons by means of neutron monitors. In order to avoid or, at least, to minimise these errors, a detailed numerical simulation of the atmospheric cascade process should be carried out. Even though the errors still exist, the records of NM responses to SNEs are of great value for solar physics.

Note that high-altitude equatorial monitors are best suited for the study of solar neutrons, while highlatitude and polar stations are more effectively used for the research of cosmic rays. Thus, both equatorial and polar NMs combined with space-borne instrumentation can provide important information on the processes of particle acceleration, propagation and interactions in solar flares.

Acknowledgements. We are grateful to Dr. L. G. Kocharov for his comments on the paper. We thank the referee for valuable comments and the Academy of Finland and the University of Oulu for financial support.

Topical Editor, R. Schwenn thanks E. O. Flückiger for his help in evaluating this paper. 


\section{References}

Belov, A. V., L. I. Dorman, V. N. Ishkov, R. A. Saidaliev, and A. G. Zusmanovich, Variations of secondary neutrons during powerful solar flares, Proc. 20th Int. Cosmic Ray Conf., Moscow, 3, 90, 1987.

Chupp, E. L., H. Debrunner, E. Flückiger, D. J. Forrest, F. Golliez, G. Kanbach, W. T. Vestrand, J. Cooper, and G. Share, Solar neutron emissivity during the large flare on 1982 June 3, Astophys.J., 318, 913, 1987.

Debrunner, H., Neutron monitor measurements as a complement to space measurements of energetic solar particle fluxes, in: High Energy Solar Phenomena, AIP Conf. Proc. \#294, Eds. J.M.Ryan and W.T.Westrand, 207, 1994.

Debrunner, H., E. Flückiger, E. L. Chupp, and D. J. Forrest, The solar cosmic ray event on June 3, 1982, Proc.18th Int. Cosmic Ray Conf., Bangalore, 4, 75, 1983.

Debrunner, H., E. Flückiger, and M. Schubnell, Present and future activities of the Bern cosmic ray group in the field of solar neutrons, in: Processes on the Surface and in the Interior of the Sun, Ed. G.E.Kocharov, $₫$ Leningrad, 9, 1987.

Debrunner, H., E. O. Flückiger, and P. Stein, On the sensitivity of neutron monitors to solar neutrons, Nucl. Instr. and Meth., A278, 573, 1989.

Debrunner, H., E. O. Flückiger, and P. Stein, On the sensitivity of a NM-64 standard neutron monitor at sea level to solar neutrons in dependence of the angular distance of the station from the sub-solar point, Proc. 21st Int. Cosmic Ray Conf., Adelaide, 5, 129, 1990.

Debrunner, H., J. A. Lockwood, and J. M. Ryan, Solar neutron and proton production during the 1990 May 24 Cosmic-ray Flare Increase, Astrophys.J., 409, 822, 1993.

Efimov, Yu. E., and M. M. Terekhov, Integral multiplicities of generation of neutron supermonitors for solar neutrons, Geomagn. and Aeronom., 28, 713, 1988.

Efimov, Yu. E., G. E. Kocharov, and K. Kudela, On the solar neutron observation on high mountain neutron monitors, Proc. 18th Cosmic Ray Conf., Bangalore, 10, 276, 1983.

Efimov, Yu. E., G. A. Kovaltsov, G. E. Kocharov, L. G. Kocharov, and I. G. Usoskin, Registration of solar neutrons by the World Neutron Monitor Network, (in Russian), Izvestiya RAN, ser.fyz., 57, N7, 142, 1993.

Evenson, P., P. Meyer, and K. R. Pyle, Protons from the decay of solar flare neutrons, Astrophys.J., 274, 875, 1983.

Gueglenko, V. G., Yu. E. Efimov, G. E. Kocharov, G. A. Kovaltsov, N. Z. Mandzhavidze, M. M. Terekhov, and L. G. Kocharov, Neutrons and gamma-ray emission on 1982 June 3: the possibility of fitting the data on the assumption of one population of accelerated particles, Astrophys.J. Suppl., 73, 209, 1990a.

Gueglenko, V. G., G. E. Kocharov, G. A. Kovaltsov, L. G. Kocharov, and N. Z. Mandzhavidze, Generation of high-energy neutral radiation in flare loops, Solar Phys., 125, 91, 1990 b.

Hatton, C. J., The Neutron Monitor, Prog. Elem. Part. Cosmic Ray Phys., 10, 1, 1971.

Hatton, C.J., and H. Carmichael, Experimental investigation of the NM-64 Neutron Monitor, Can.J.Phys., 42, 2443, 1964.

Hudson, D., Statistics: Lectures on Elementary Statistics and Probability, $₫$ Geneva, 1964.
Iucci, N., M. Parisi, C. Signorini, M. Storini, C. Villoresi, and N. L. Zangrilli, The possibility of observing solar neutrons events in the lower atmosphere, Nouvo Cimento, 7C, 732, 1984.

Kocharov, L. G., J. W. Lee, H. Zirin, G. A. Kovaltsov, I. G. Usoskin, K. R. Pyle, M. A. Shea, and D. F. Smart, Neutron and electromagnetic emission during the 1990 May 24 solar flare, Solar Phys., 155, 149, 1994

Kocharov, L. G., G. A. Kovaltsov, J. Torsti, and R. Vainio, Search for neutron decay protons from the 1990 May 24 solar flare, Proc. 24th Int. Cosmic Ray Conf., Rome, 4, 163, 1995.

Kovaltsov, G. A., Yu. E. Efimov, and L. G. Kocharov, Ground-level energetic solar particle event on 24 May 1990: possibility of direct solar neutron detection, Solar Phys. Lett., 144, 195, 1993.

Kovaltsov, G. A., L. G. Kocharov, I. G. Usoskin, H. Kananen, P. Tanskanen, and A. Yu. Drobysh, Two components of highenergy neutrons during the solar flare of May 24, 1990, Izvestiya $R A N$, ser.fyz., 59, N4, 195, 1995a.

Kovaltsov, G. A., L. G. Kocharov, I. G. Usoskin, H. Kananen, and P. J. Tanskanen, Two injections of high-energy neutrons during the May 24, 1990 solar flare, Proc. 24th Int. Cosmic Ray Conf., Rome, 4, 155, 1995 b.

Mandzhavidze, N. Z., and R. Ramaty, High-energy gamma-ray emission from pion decay in solar flare magnetic loop, Astrophys.J., 389, 739, 1992.

Muraki, Y., S. Sakakibara, S. Shibata, K. Murakami, K. Takahashi, K. R. Pyle, T. Sakai, K. Mitsui, M. A. Shea, and D. F. Smart, Simultaneous observations of solar neutrons on June 6, 1991 at Mt.Haleakala and Mt.Norikura, Proc. 24th Int. Cosmic Ray Conf., Rome, 4, 175, 1995.

Ramaty, R., and R. J. Murphy, Nuclear processes and accelerated particles in solar flares, Space Sci.Rev., 45, 213, 1987.

Pyle, K. R., The Haleakala cosmic ray neutron monitor station: intercalibration with Huancayo station, Proc. 23rd Int. Cosmic Ray Conf., Calgary, 3, 609, 1993.

Shea, M. A., and D. F. Smart, A summary of major solar proton events, Solar Phys., 127, 297, 1990.

Shea, M. A., D. F. Smart, and K. R. Pyle, Direct solar neutrons detected by neutron monitors on 24 May 1990, Geophys. Res. Lett., 18, 1655, 1991.

Shibata, S., Propagation of solar neutrons through the atmosphere of the earth, J.Geophys. Res., 99, 6651, 1994.

Smart, D. F., M. A. Shea, and K. O'Brien, Comment on the minimum mass path of a uni-directional solar neutron particle beam through the Earth atmosphere, Proc. 24th Int. Cosmic Ray Conf., Rome, 4, 171, 1995.

Stoker, P. H., Search for solar neutron recording by the 18NM64 at Tsumeb during the 21st Solar Cycle, Proc.20th Int. Cosmic Ray Conf., Moscow, 3, 98, 1987.

Stoker, P. H., Relativistic solar proton events, Space Sci.Rev., 73, 327, 1994.

Takahashi, K., Detection of solar neutrons by ground-based neutron monitor, Space Sci. Rev., 51, 123, 1989.

Takahashi, K., M. Wada, M. Yoshimori, M. Kusunose, and I. Kondo, Search for solar neutrons using NM64 equipments, Proc.20th Int. Cosmic Ray Conf., Moscow, 3, 82, 1987.

Zusmanovich, A. G., and Ya. E. Shwartsman, Solar cosmic rays at the middle latitudes, in: Processes on the Surface and in the Interior of the Sun, Eds. G. E. Kocharov, Leningrad, 1987. 